\title{
El mercado de trabajo en Andalucía y Cataluña desde la perspectiva de género. Su evolución durante el período de 1995 a 2005
}

\author{
Marta Ortega Gaspar \\ Universidad de Málaga \\ maga@uma.es
}

Recibido: 24-07-2008

Aceptado: 15-05-2009

\section{Resumen}

El presente estudio muestra el contraste existente entre dos regiones españolas, Andalucía y Cataluña, en el mercado de trabajo desde una doble perspectiva de tiempo y de género. Los datos permiten otorgar a Cataluña una posición más cercana a una modernización teórica, siempre dentro del plano laboral aquí estudiado, si bien es cierto que estos datos permiten observar, para el caso concreto de Andalucía, un constante acercamiento al proceso de modernización. Las ideas centrales que se sostienen son dos: la primera es que, a medida que una sociedad avanza, crece en igualdad entre sus miembros (que en este análisis se ha focalizado en la desigualdad de sexo en el mercado de trabajo); la segunda consiste en mantener la tesis de que dicho proceso de modernización genera, a su vez, un creciente desarrollo de individuación, es decir, que las categorías sociales que en las sociedades premodernas eran útiles para posicionar socialmente a sus individuos, dejan de serlo en la sociedad moderna.

Palabras clave: cambio social, modernización, desigualdad social, mercado de trabajo, género, desigualdad entre comunidades autónomas.

Abstract. The labour market in Andalusia and Catalonia from a gender perspective. The evolution from 1995 to 2005

This study presents the contrast between two Spanish regions, Andalusia and Catalonia, in the context of the labour market, from a double perspective: time and gender. The results situated Catalonia in a more modern position than Andalusia, within in the labour context that is the main focus of this analysis. Although it must be emphasised the constant change that is taking place in Andalusia towards modernity. The main ideas that central in the whole study are: on the one hand, that a more modern society implies a more equalitarian relation among its members, and on the other hand, that the process of modernity is followed by an increasing process of individualization, meaning that all those categories that were useful in pre-modern societies to position socially its individuals became useless in modern society.

Key words: social change, modernity, social inequality, labour market, gender, region inequalities. 


\section{Sumario}

1. Objetivos y antecedentes

2. Metodología

3. Indicadores estructurales de desigualdad de género en el trabajo
4. Factores personales y mercado de trabajo

5. Conclusiones

Referencias bibliográficas

Anexo

\section{Objetivos y antecedentes}

El objetivo del presente trabajo es conocer la evolución vivida en Andalucía y en Cataluña en el mercado laboral en el período que se extiende de 1995 a 2005 , desde la perspectiva de género ${ }^{1}$. La hipótesis de partida defiende la idea de que el mercado laboral es más moderno en la medida en la que hay más igualdad de oportunidades. En el caso que nos ocupa, es más moderno cuando existe igualdad de oportunidades para los hombres y las mujeres en el mercado de trabajo. Se entiende que, a más modernización, más igualdad de oportunidades para todos $y$, por lo tanto, también de género.

El concepto de modernización (transformación de la estructura social y cómo ésta afecta a la vida y al comportamiento de sus miembros) ${ }^{2}$ aquí utilizado comprende dos desarrollos básicos: el económico o incremento del PIB per cápita y el social o aumento de la individuación. A estos desarrollos, se pueden añadir otros que suelen acompañar a los anteriores, pero que aquí no han sido considerados: el cultural, o incremento de valores individualistas; el político, o aceptación de la democracia del ciudadano, y, entre otros, el espacial, o incremento de las vías de comunicación de ideas, personas y cosas. Este trabajo se ha centrado en operativizar la dimensión del desarrollo social, entendiéndose por éste el proceso mediante el cual el individuo se independiza cada vez más de las categorías sociales de origen e incluso de las categorías sociales de pertenencia ${ }^{3}$. Se constata que la modernización dificulta la explicación de la conducta de los sujetos a través de las categorías sociales de los mismos y que,

1. El presente trabajo queda enmarcado en el estudio sobre el cambio social en Andalucía subvencionado por la Fundación Centro de Estudios Andaluces (Ref. SOCH 1.07/023), entidad dependiente de la Consejería de la Presidencia de la Junta de Andalucía.

2. El concepto de modernización es uno de los más confusamente utilizados en la sociología contemporánea. Surge a medida que la corriente neoevolucionista incide en la formulación de las categorías del estructural-funcionalismo para tratar de explicar el cambio social de las sociedades avanzadas, modernas, en el período que sigue a la Segunda Guerra Mundial. Bajo el supuesto de que la modernización es un proceso, el cambio se refiere a las transformaciones por diferenciación estructural y reintegración de las partes (instituciones) que componen una sociedad entre los extremos de un continuo. Las definiciones de modernización de los años setenta tienen en cuenta la transformación estructural de la sociedad y cómo ésta afecta directamente a la vida de sus miembros (Giner, Lamo de Espinosa y Torres, 1998: 498-500). Véase Bernstein (ed.) (1985) y Giddens (1991).

3. Gobernado Arribas (1996a: 8). 
en el caso que nos ocupa, a mayor grado de modernización de una sociedad, menor posibilidad de que el sexo condicione los comportamientos de sus miembros.

En este trabajo, se parte del supuesto de que las diferencias de comportamientos en el mercado de trabajo son producto de tres tipos de factores: $a$ ) personales, $b$ ) de la estructura productiva y $c$ ) del sistema de políticas sociales y de empleo de cada región. En el análisis que aquí se presenta, se contemplan los factores personales que contribuyen a perfilar el mercado laboral de las mujeres y, sobre todo, las diferencias entre las mujeres casadas que trabajan y las que no, en los dos contextos contemplados.

Concretamente, las preguntas a las que se ha dado respuesta son las siguientes: ¿Cuál es la situación actual en el mercado laboral de las mujeres y de los hombres en Andalucía y en Cataluña? ¿La posición social del esposo o compañero ejerce influencia sobre la situación ocupacional de la mujer casada o que vive en pareja? ¿Cuál es la probabilidad de que la mujer casada trabaje fuera de casa? ¿Existen diferencias entre las dos regiones estudiadas?

Es evidente el cambio constante y acelerado que acecha a la sociedad occidental en la actualidad, y específicamente a la sociedad española. En España, entre los cambios sociales vividos más recientemente en el ámbito laboral, destaca el experimentado por las féminas y, sobre todo, por las mujeres casadas y madres de familia. Estos cambios producen, a su vez, transformaciones en las relaciones laborales entre los hombres y las mujeres e invitan a la reflexión sobre la desigualdad de género en dicho terreno. Bericat y Sánchez (2008) indican que el gran cúmulo de transformaciones experimentadas durante el siglo XX sólo puede comprenderse analizando la específica interacción de vectores tecnológicos y sociales, pues sólo así podrá explicarse la paradoja de que distintas sociedades hayan alcanzado muy diferentes estadios de desarrollo, aún cuando el conocimiento científico y técnico disponible sea bastante similar. Los autores citados entienden que uno de los vectores de cambio social y cultural más importantes de todo el siglo XX es el proceso mediante el cual las sociedades han pretendido alcanzar la plena igualdad de género ${ }^{4}$.

Como ya se ha indicado, el foco central de análisis es el cambio experimentado por las dos comunidades ya referidas en el terreno laboral. Para ello, se parte de la idea de que hay dos tipos de desigualdad (Gobernado, 1996c): la que proviene del capital humano acumulado individualmente (conocimien-

4. Establecen un sistema de indicadores de desigualdad para medir, de forma válida y fiable, en qué grado avanza la sociedad, cuál es el ritmo con el que avanza y en qué dimensiones particulares avanza. En este trabajo, se utilizarán algunos de estos índices que permitirán articular el cambio experimentado en el plano laboral en las dos comunidades autónomas aquí estudiadas. Dichos indicadores sociales permiten obtener un índice sintético de desigualdad de género, que resume en una sola cifra este importante rasgo de la realidad social. 
tos, habilidades, etc.) y la que proviene del capital acumulado colectivamente (por ser mujer, de familia acomodada, por haber nacido en una comunidad autónoma determinada, etc.). Se mantiene la tesis de que la sociedad moderna conlleva un menor grado de desigualdad proveniente de la acumulación de capital colectivo, en cambio, predomina la desigualdad originada en el capital individual acumulado. Así, al comparar dos sociedades, se entenderá que aquélla en la que el prestigio social de sus miembros (hombres y mujeres) tenga menos que ver con las categorías sociales que aluden al sexo, al estado civil, al origen de clase, al hábitat de nacimiento, etc., es decir, al capital colectivo, serán más modernas o avanzadas que aquéllas otras donde la influencia de aquél sea superior ${ }^{5}$.

Nuestro propósito es conocer la desigualdad de género en el terreno laboral según el estatus social del cónyuge o de la pareja masculina (capital acumulado colectivamente). Se analiza la desigualdad de posición sociolaboral utilizando un índice de prestigio ocupacional (Carabaña y Gómez Bueno, 1996; Gobernado, 1996b; Montoro, 2003). Es decir, las variables a considerar son el prestigio ocupacional del marido y la situación de la mujer en el mercado laboral.

El contexto socioeconómico general en el que tiene lugar este análisis es el de la sociedad española, que vive importantes transformaciones. Entre otras, ha sufrido el cambio del mercado laboral como consecuencia de la creciente incorporación de la mujer al trabajo asalariado, concretamente de la mujer casada ${ }^{6}$. El estudio, como se ha repetido ya, se centra en el análisis comparado de la realidad social existente en este punto en Andalucía y Cataluña.

El incremento del número de mujeres casadas trabajadoras hace surgir un proceso denominado polarización social (Esping-Andersen et al., 2005), un proceso que, aparentemente, es positivo y funcional para todo el sistema social, pues los dobles ingresos en el hogar dificultan la caída de la pobreza, tiende a convertirse en disfuncional, al contribuir a perpetuar las desigualdades vigentes cuando la participación en el mercado laboral de las mujeres se concentra en los hogares donde ambos cónyuges tienen un alto nivel educativo ${ }^{7}$. De esa manera, se refuerza y se mantiene la estructura de desigualdad ya existente en nuestra sociedad (Salido, 2007).

5. Ha de entenderse, como bien explica Gobernado Arribas (1996c), que, en una supuesta sociedad totalmente moderna, seguirá existiendo desigualdad social (de ingresos, de prestigio, etc.), pero lo peculiar del caso es que tal desigualdad provendrá principalmente del capital acumulado individualmente (esfuerzo, conocimientos, etc.).

6. Además, la trayectoria laboral de las mujeres casadas ha tendido a homogeneizarse con la de los hombres casados, como ya observó, entre otros, Tobío (1994).

7. El acceso a los estudios, especialmente a los de nivel postsecundario (técnicos, profesionales, universitarios) incide de manera positiva en el camino hacia una sociedad donde exista mayor igualdad entre los sexos, no sólo en el ámbito educativo, sino también en el familiar y laboral (Mayoral y Samper, 2006: 39 y Garrido, 1992: 187). 


\section{Metodología}

En la elaboración de la descripción del panorama laboral según el sexo en Andalucía y Cataluña, el universo poblacional utilizado ha sido el compuesto por los hombres y las mujeres en edad de trabajar que viven en dichos territorios. En la segunda parte del trabajo, este universo ha sido restringido a los hombres y a las mujeres ocupados, casados o que viven como si lo estuvieran y que residen en Andalucía y en Cataluña (es decir, este es el universo poblacional utilizado para contestar a la pregunta de si existe relación entre el prestigio ocupacional del esposo o compañero y la situación ocupacional de las mujeres). Además, ha de señalarse que los datos obtenidos han sido comparados con los resultados hallados para el total nacional.

Las fuentes de datos estadísticos utilizadas han sido: la Encuesta de Población Activa (EPA, INE) segundos trimestres ${ }^{8}$ de 1995 y 2005; la Encuesta de Calidad de Vida en el Trabajo (ECVT, Ministerio de Trabajo y Asuntos Sociales) desde 1999 hasta 2004 (estos datos han sido tratados de forma agrupada) y el Panel de Hogares de la Unión Europea, año 2000 (PHOGUE, 2000).

Para estudiar la relación entre la situación profesional de la mujer casada y el prestigio ocupacional del marido, se ha recodificado la variable situación profesional del cónyuge o de la pareja incluida en la ECVT (1999-2004) de la siguiente manera. Las mujeres casadas o que viven como si lo estuvieran que se encontraban trabajando en cualquier tipo de situación de todas las recogidas en la citada encuesta, son agrupadas en la categoría «trabaja fuera de casa». En la categoría «no trabaja fuera de casa», quedan recogidas las amas de casa, las jubiladas y las estudiantes. La categoría de «desempleado» no ha sido tenida en cuenta en esta clasificación. El prestigio ocupacional se ha medido siguiendo las puntuaciones establecidas por Carabaña y Gómez Bueno (1996: 55 y s.) y queda recogido en la variable de prestigio ocupacional o PRESCA2. La variable PRESCA2 recoge las puntuaciones que los entrevistados dan a las ocupaciones con referencia al modelo «Dependiente de comercio al que se le atribuye la puntuación 100», con valores de 0 en adelante y sin límites en las puntuaciones admisibles. De ello resulta una escala de razón.

Con el fin de conocer los determinantes de la probabilidad de que la mujer casada trabaje fuera de casa según su edad, el nivel de estudios personal y de su marido, la tenencia de hipoteca, la atención dedicada al cuidado de niños, la atención dedicada al cuidado de adultos dependientes, la situación profesional del marido y los ingresos de éste, se han estimado tres modelos logit ${ }^{9}$ : uno de ellos recoge los datos del conjunto español y los otros dos se han centrado en cada una de las poblaciones objeto del presente estudio: la andaluza

8. Es norma utilizar el segundo trimestre de cada año de la EPA, al considerar su mayor representatividad del conjunto del año, bajo el supuesto de que se minimizan los factores estacionales y la inclusión, en su cuestionario, de las denominadas preguntas retrospectivas.

9. La razón por la que se ha aplicado un logit para el análisis de los datos anteriormente expuestos es que la variable «dependiente» es una variable nominal o categórica. 
y la catalana. En este análisis, como se indicó previamente, se han utilizado los datos del Panel de Hogares de la Unión Europea (PHOGUE, 2000) ${ }^{10}$. La variable dependiente se ha dividido en dos categorías: «trabaja fuera de casa» y "no trabaja fuera de casa».

\section{Indicadores estructurales de desigualdad de género en el trabajo}

\subsection{Relación con la actividad económica según el sexo y la comunidad autónoma. Andalucía y Cataluña 1995-2001 y 2002-2005}

En el siguiente análisis, los datos han sido desagregados en los intervalos de tiempo que se extienden desde 1995 hasta 2001, 2002 y 2005, para que las comparaciones se ajustaran a los criterios conceptuales y estadísticos que en cada momento se han utilizado en la Encuesta de Población Activa, realizada por el Instituto Nacional de Estadística. La Encuesta de Población Activa (EPA) se modificó en $2002^{11}$.

Lo primero a destacar son las diferencias estructurales de los dos ámbitos objeto de estudio (Andalucía y Cataluña), que se materializan a través de indicadores básicos del mercado de trabajo como la tasa de actividad, de empleo y de paro, aquí analizados.

En Cataluña, en 2005, según los datos de la EPA del segundo trimestre de 2005, existe una tasa de actividad que se sitúa en el 75,68\% (en Andalucía es del 65,27\%). La tasa de empleo en Cataluña es del 70,25\% y para Andalucía

10. La elección del PHOGUE 2000 no es arbitraria. Se ha escogido esta encuesta porque permite tener información tanto de las mujeres que trabajan como de las que no y además ofrece información sobre los datos de parentesco, de tal manera que conseguimos conocer como afectan ciertas características de los maridos o compañeros sobres sus mujeres o compañeras sentimentales.

Hay que hacer constar que, al fichero de adultos, se le ha añadido el fichero de relaciones de parentesco. En esta parte del trabajo, así como para el desarrollo de los modelos logit, ha sido crucial la labor realizada por el profesor Carlos Gamero, del Departamento de Estadística de la Universidad de Málaga. También la encuesta PHOGUE 2000 permite obtener submuestras representativas a nivel de las comunidades autónomas, al haberse ampliado la muestra habitual.

11. Los cambios experimentados por la EPA en la fecha indicada son de carácter metodológico y por ello afectan tanto a las definiciones utilizadas en la encuesta, como al proceso de cálculo de los resultados. Estas transformaciones son el resultado de: 1. la utilización de nuevas proyecciones de población del INE (hecho originado por el aumento de población experimentado en España como consecuencia de la llegada de inmigrantes); 2. una mejora técnica en el cálculo de los resultados de la encuesta (que corrige el efecto de la falta de respuesta en la encuesta); 3. la aplicación de la nueva definición de paro establecida en el Reglamento (CE) número 1897/2000 de la Comisión, de 7 de septiembre de 2000 (este último cambio contribuye a la homogeneización en la recogida de los datos sobre el mercado de trabajo a nivel comunitario, lo cual facilita la labor de la investigación social comparada). De las tres modificaciones técnicas, la nueva definición de parado (que, aunque sigue vigente la definición internacional de la OIT, introduce cambios en las instrucciones sobre cómo interpretar la búsqueda activa de trabajo) es la que tiene mayor efecto en las cifras de paro, lo cual provoca una disminución en las cifras del desempleo, tanto absolutas como relativas. 
Tabla 1. Evolución de la tasa de actividad de 1995 a 2005. Dato comparativos de la población de 16 a 64 años por sexos y comunidad autónoma

\begin{tabular}{lrrrrrr}
\hline $\begin{array}{l}\text { Tasa de } \\
\text { actividad \% }\end{array}$ & $\mathbf{1 9 9 5}$ & $\mathbf{2 0 0 1}$ & $\begin{array}{c}\text { Diferencia } \\
\text { b-a }\end{array}$ & $\mathbf{2 0 0 2}$ & $\mathbf{2 0 0 5}$ & $\begin{array}{c}\text { Diferencia } \\
\text { b-a }\end{array}$ \\
\hline Andalucía & & & & & & \\
Ambos sexos & 49,37 & 49,47 & 0,10 & 52,32 & 65,27 & 12,95 \\
Hombres & 64,70 & 63,93 & $-0,77$ & 66,08 & 78,79 & 12,71 \\
Mujeres & 34,84 & 35,69 & 0,85 & 39,19 & 51,55 & 12,36 \\
Diferencia M-H & $-29,86$ & $-28,24$ & $-1,62$ & $-26,84$ & $-27,24$ & $-0,35$ \\
\hline Cataluña & & & & & & \\
Ambos sexos & 54,01 & 58,15 & 4,14 & 58,38 & 75,68 & 17,30 \\
Hombres & 66,76 & 70,26 & 3,50 & 70,21 & 85,61 & 15,40 \\
Mujeres & 41,99 & 46,67 & 4,68 & 47,14 & 65,52 & 18,38 \\
Diferencia M-H & $-24,77$ & $-23,59$ & 1,18 & $-23,07$ & $-20,09$ & 2,98 \\
\hline
\end{tabular}

Fuente: Encuesta de Población Activa, Instituto Nacional de Estadística (INE). Datos referidos a los segundos trimestres de cada año.

del $56,23 \%$, y la de paro, del $7,17 \%$ y del $13,84 \%$, respectivamente y para la misma fecha antes referida. Es decir, las diferencias entre estas comunidades en cuanto a la actividad son de diez puntos porcentuales. Respecto al empleo, la diferencia es incluso mayor, concretamente, de 14,02 puntos porcentuales. Y en lo que concierne a la tasa de paro, Andalucía tiene una tasa casi el doble mayor que la de Cataluña. Todo ello puede verse en las tablas 1,2 y 3.

En Cataluña, la diferencia entre mujeres y hombres respecto a la tasa de actividad decrece más rápido que en Andalucía, porque, entre otras razones, en Cataluña la tasa de actividad femenina sufre un incremento superior a la masculina. Dicho crecimiento es de casi tres puntos porcentuales en Cataluña, mientras que en Andalucía, aunque también se vive un proceso de crecimiento de la tasa de actividad entre las mujeres, dicho crecimiento sigue siendo inferior al de la tasa de actividad masculina, con una diferencia de 0,35 puntos porcentuales favorable a los varones.

Otra observación interesante es que, en lo relativo a la tasa de actividad y para ambos sexos, Andalucía y Cataluña ofrecen unas tasas superiores a la media nacional. Aunque volvemos a insistir en que Cataluña se distancia con creces de la media española, tanto en el caso de los varones (con una tasa de diecisiete puntos porcentuales superior a la media) como en el de las mujeres (con una tasa de diecinueve puntos porcentuales por encima de la media de las mujeres españolas).

Es de subrayar que este fenómeno es más reciente en el caso de Andalucía, pues, como indica Castaño (2002), Cataluña, en 2001, ya presentaba unas tasas de actividad cercanas al 60\% (doce puntos porcentuales por encima de la media española). Sin embargo, Andalucía tenía una tasa de actividad en torno al 47\%, con lo cual, en 2001, ambas comunidades estaban separadas por una amplitud o distancia de 13 puntos porcentuales. En la comparación realizada entre el conjunto de las comunidades autónomas en España, sus tasas 
Tabla 2. Evolución de la tasa de empleo de 1995 a 2005. Datos comparativos de la población de 16 a 64 años por sexo y comunidad autónoma

\begin{tabular}{|c|c|c|c|c|c|c|}
\hline \multirow{2}{*}{$\begin{array}{l}\text { Tasa de } \\
\text { empleo }\end{array}$} & \multicolumn{5}{|c|}{ Diferencia } & \multirow{2}{*}{$\begin{array}{c}\text { Diferencia } \\
\text { b-a }\end{array}$} \\
\hline & 1995 & 2001 & & 2002 & 2005 & \\
\hline \multicolumn{7}{|l|}{ Andalucía } \\
\hline Ambos sexos & 32,94 & 40,49 & 7,55 & 42,45 & 56,23 & 13,78 \\
\hline Hombres & 46,63 & 55,37 & 8,74 & 57,34 & 70,44 & 13,10 \\
\hline Mujeres & 19,98 & 26,31 & 6,33 & 28,24 & 41,83 & 13,59 \\
\hline Diferencia M & $-26,65$ & $-29,06$ & $-2,41$ & $-29,1$ & $-28,61$ & 0,49 \\
\hline \multicolumn{7}{|l|}{ Cataluña } \\
\hline Ambos sexos & 43,34 & 53,24 & 9,90 & 52,79 & 70,25 & 17,25 \\
\hline Hombres & 56,61 & 65,77 & 9,16 & 65,27 & 80,44 & 15,17 \\
\hline Mujeres & 30,84 & 41,37 & 10,53 & 40,95 & 59,83 & 18,88 \\
\hline Diferencia M & $-25,77$ & $-24,40$ & 1,37 & $-24,32$ & $-20,61$ & 3,71 \\
\hline
\end{tabular}

Fuente: Encuesta de Población Activa, Instituto Nacional de Estadística (INE). Datos referidos a los segundos trimestres de cada año.

representaban el máximo regional en el caso de Cataluña y el mínimo en el de Andalucía.

En 2005, la distancia entre las tasas de actividad de Cataluña y Andalucía es de algo más de diez puntos porcentuales, con lo que se ha producido un ligero acercamiento en este terreno entre ambas comunidades. En 2005, en términos medios para el conjunto nacional, la tasa de actividad de la mujer es del 46,49; para Cataluña, del 65,52 y para Andalucía, del 51,55, con lo que ambas comunidades cuentan con una tasa de actividad superior a la media nacional. En este punto, cabe considerar el papel desempeñado, tanto por el aumento de la formación entre la población femenina española, como por el considerable incremento de la población inmigrante en las dos regiones aquí analizadas.

Si nos detenemos en la evolución de este indicador, la tasa de actividad femenina desde 2002 hasta 2005, se aprecia que el cambio más agudo lo han vivido las mujeres catalanas, pues los niveles de participación femenina han experimentado un crecimiento de 18,38 puntos porcentuales en el período referido. En el caso de Andalucía, el crecimiento ha sido de 12,36 puntos porcentuales.

Los cambios sufridos en el empleo han sido considerables en ambas comunidades, y han supuesto en ambos casos importantes crecimientos en las tasas de empleo. La desagregación del período analizado (de 1995 a 2005) en los dos intervalos de tiempo previamente indicados (de 1995 a 2001 y de 2002 a 2005) permite observar que, en la segunda etapa, el crecimiento del empleo se acelera en ambas comunidades. El cambio que se vive en los cuatro años que distan entre 2002 y 2005 es más pronunciado que el vivido entre 1995 y $2001^{12}$.

12. Si bien no se ha de olvidar que esta comparación ha de realizarse con cautela, dados los cambios conceptuales y metodológicos realizados por el INE en la elaboración de la EPA a partir de 2002. 
La diferencia sufrida por la tasa de empleo entre 2002 y 2005 en Cataluña es casi del doble que la experimentada entre 1995 y 2001. Y en Andalucía la diferencia es bastante similar, aunque algo menos pronunciada.

Otro hecho destacable es el que hace referencia a las transformaciones experimentadas en el empleo según el sexo en ambas comunidades. En la tabla 4, se aprecia que Cataluña experimenta los cambios que aluden a las diferencias entre los sexos a nivel de empleo antes que Andalucía. En otras palabras, se observa que Cataluña se sube a la reciente tendencia vivida en el mercado laboral antes que Andalucía. Dicha nueva tendencia no es otra que el mayor crecimiento generalizado del empleo femenino, que tiende a superar al crecimiento del empleo masculino.

Así, se constata que en Cataluña, en el período que se extiende de 1999 a 2001, la diferencia entre mujeres y hombres en la tasa de empleo es ya favorable a las primeras (con una distancia entre los sexos de 1,37), mientras que en Andalucía, en el mismo período, el crecimiento de la tasa de empleo masculino sigue siendo superior al crecimiento de la tasa de empleo femenino, por lo que la diferencia entre mujeres y hombres es desfavorable para las féminas $(-2,41)$. Aunque creo conveniente insistir que este cambio se vive dentro de un contexto de expansión del empleo para ambos sexos.

En el período que abarca desde 2002 hasta 2005, en Cataluña, las diferencias entre las tasas de empleo de las mujeres y de los hombres es favorable a las primeras. Esta distancia es de 3,71 puntos porcentuales. Es decir, aunque la tasa de empleo femenino en Cataluña sigue siendo inferior a la masculina, el crecimiento que vive el empleo femenino ofrece un ritmo más rápido que el de los hombres.

En Andalucía, dentro del mismo intervalo de tiempo, comienza a observarse esta nueva tendencia (de mayor aceleración del crecimiento del empleo entre las féminas), aunque aún los datos indican que en esta región todavía se trata de un ligero despegue, concretamente, la diferencia entre las tasas de empleo de las mujeres y de los hombres es de 0,49 puntos porcentuales favorables a las primeras.

Vuelvo a insistir en el hecho de que la mayor aceleración en el crecimiento del empleo femenino respecto al masculino puede interpretarse como una tendencia o un indicador de modernidad, si entendemos por ésta un caminar hacia una sociedad donde la igualdad entre los sexos sea posible.

Otro indicador que he considerado en el análisis de los cambios habidos en las desigualdades entre los sexos en el mercado de trabajo en Andalucía y Cataluña es el de la tasa de paro.

Como anteriormente se indicó, las desigualdades entre los sexos en las cuestiones relativas al desempleo son más acusadas en las sociedades menos avanzadas, en las que es fácil observar cómo las mujeres suelen padecer en mayor medida que los hombres las mayores dificultades para acceder a un trabajo remunerado. Este fenómeno queda explicado tanto por razones económicas como culturales, pues la tendencia observada es la de que, en aquellas sociedades en las que escasea el trabajo, se tiende a primar el empleo del varón, 
Tabla 3. Evolución de la tasa de paro de 1995 a 2005. Datos comparativos de la población de 16 a 64 años por sexo y comunidad autónoma

\begin{tabular}{lcrrrrr}
\hline $\begin{array}{l}\text { Tasa } \\
\text { de paro }\end{array}$ & $\mathbf{1 9 9 5}$ & $\mathbf{2 0 0 1}$ & $\begin{array}{c}\text { Diferencia } \\
\text { b-a }\end{array}$ & $\mathbf{2 0 0 2}$ & $\mathbf{2 0 0 5}$ & $\begin{array}{c}\text { Diferencia } \\
\text { b-a }\end{array}$ \\
\hline $\begin{array}{l}\text { Andalucía } \\
\text { Ambos sexos }\end{array}$ & 33,28 & 18,15 & $-15,13$ & 18,87 & 13,84 & $-5,03$ \\
Hombres & 27,94 & 13,38 & $-14,56$ & 13,23 & 10,60 & $-2,63$ \\
Mujeres & 42,66 & 26,29 & $-16,37$ & 27,94 & 18,87 & $-9,07$ \\
Diferencia M - H & 14,72 & 12,91 & 1,81 & 14,71 & 8,27 & 6,44 \\
\hline Cataluña & & & & & & \\
Ambos sexos & 19,75 & 8,44 & $-11,31$ & 9,57 & 7,17 & $-2,40$ \\
Hombres & 15,22 & 6,39 & $-8,83$ & 7,04 & 6,04 & $-1,00$ \\
Mujeres & 26,54 & 11,36 & $-15,18$ & 13,14 & 8,69 & $-4,45$ \\
Diferencia M- H & 11,32 & 4,97 & 6,35 & 6,10 & 2,65 & 3,45 \\
\hline
\end{tabular}

Fuente: Encuesta de Población Activa, Instituto Nacional de Estadística (INE). Datos referidos a los segundos trimestres de cada año.

reflejo del predominio de una cultura patriarcal (división del trabajo según el sexo de los cónyuges [Parsons, 1955]) que se rige por la división del trabajo en función del sexo. De esta manera, se tiende a situar al varón en la esfera pública, puesto que es considerado como sujeto productivo y sustentador económico principal del hogar; y a la mujer, en la esfera privada, el ámbito doméstico, puesto que es considerada como un sujeto reproductivo que ha de centrarse en las tareas de la casa.

Las diferencias habidas en las tasas de desempleo según el sexo en las dos comunidades aquí analizadas durante el período de 2002 a 2005 reflejan una menor desigualdad entre los sexos en Cataluña (un 3,45\% frente a un 6,44\% en Andalucía). Por otra parte, es de destacar que, en ese mismo período, el desempleo femenino en Andalucía decrece en mayor proporción que en Cataluña (en la primera, la disminución es de 9,07 puntos porcentuales y, en Cataluña, de 4,45 puntos porcentuales). Es decir, decrece más en donde es más elevado, en Andalucía.

También es importante hacer notar que, en términos relativos y según los datos de la EPA correspondientes al segundo trimestre de 2005, Cataluña vuelve a situarse en una mejor posición que la media nacional, puesto que la tasa de desempleo femenino en esta región es bastante inferior a la media española. Por el contrario, Andalucía (con un 18,87\%) ofrece una tasa de desempleo femenino superior en algo más de seis puntos porcentuales a la media de España (que se sitúa en un 12,22\%). Como indica Castaño (2002: 274), entorno al desempleo también existe una elevada diferenciación regional. Destacan Andalucía y Extremadura en el extremo que ofrece los más altos niveles ${ }^{13}$.

13. Los datos analizados son de los segundos trimestres de la EPA de 1994 y 2001. La misma autora destaca la incidencia de la variable «nivel de estudios» en las tasas de desempleo femenino, puesto que se observan las menores tasas de desempleo para las mujeres de estudios 
Tabla 4. Tabla comparativa. Indicadores de desigualdad. Andalucía y Cataluña

\begin{tabular}{|c|c|c|c|}
\hline & \multicolumn{3}{|c|}{ Desigualdad laboral } \\
\hline & $\begin{array}{l}\text { Índice de desigualdad } \\
\text { de género laboral }\end{array}$ & $\begin{array}{c}\text { Participación laboral } \\
\text { D1 }\end{array}$ & $\begin{array}{c}\text { Condiciones laborales } \\
\text { D2 }\end{array}$ \\
\hline España & 0,281 & 0,284 & 0,278 \\
\hline Andalucía & 0,344 & 0,402 & 0,285 \\
\hline Cataluña & 0,248 & 0,221 & 0,275 \\
\hline
\end{tabular}

Fuente: Extraído de Bericat y Sánchez (2008) y elaborado, a su vez, a través de diversas fuentes.

\subsection{Los indicadores de desigualdad en Andalucía y Cataluña}

El análisis de los índices elaborados por Bericat y Sánchez (2008) nos permite posicionar ambas comunidades entre sí y respecto al conjunto nacional ${ }^{14}$.

El índice general de desigualdad laboral aporta, de forma clara y resumida, una interesante información sobre un conjunto de aspectos que comportan el ámbito laboral. Además, permite comparar con mayor facilidad las dos comunidades objeto de nuestro estudio, y de éstas con el resto de España. Dicho índice, concretamente, recoge la información de dos dimensiones de la desigualdad laboral: la que alude a la participación laboral y la que concierne a las condiciones de trabajo.

La dimensión 1, o dimensión de participación laboral, es el resultado de la suma de cuatro indicadores: la tasa de actividad y empleo de las personas entre 16 y 64 años, y la tasa de actividad y de empleo de las personas entre 16 y 44 años. La dimensión 2, o dimensión de las condiciones laborales, es el producto de cinco indicadores: los asalariados con contrato indefinido, los ocupados a tiempo completo, el salario medio y el salario medio ponderado según tipo de ocupación, y los empleados en ocupaciones con infrarrepresentación femenina.

En la tabla 4, puede contemplarse como el índice de desigualdad de género laboral (primera columna) toma el menor valor para el caso de Cataluña y, al acercarse más hacia $0^{15}$ que el resto de los valores, muestra el menor nivel

universitarios. Son también significativas las diferencias regionales en la evolución de las tasas de desempleo por niveles de estudios. Andalucía y Cataluña, según los datos de 2001, se encuentran entre las siete comunidades autónomas donde las mayores tasas de paro femenino se producen para el colectivo con estudios más bajos. Además, Andalucía y Extremadura presentan los mayores niveles de desempleo femenino en todos los niveles de estudios.

14. Los autores citados realizan un análisis de la desigualdad de género teniendo en cuenta tres aspectos de ésta: la desigualdad educativa, la laboral y la de poder. En el presente estudio, simplemente nos detenemos en observar lo que concierne al ámbito laboral para el caso concreto de Cataluña y Andalucía, teniendo en cuenta dos dimensiones: la participación laboral y las condiciones de trabajo.

15. Cuando el valor del índice es igual a 0 , no existe desigualdad de género. Cuando es positivo, mayor que cero, la desigualdad favorece a los hombres; y si es negativo, menor que cero, la desigualdad favorece a las mujeres. Para una amplia descripción del índice, véase Bericat y Sánchez (2008: 7). 
de desigualdad existente en esta sociedad frente a la mayor desigualdad laboral vivida en Andalucía. Esta última comunidad ofrece, a su vez, un nivel de desigualdad laboral mayor que el de la media española. En otras palabras, este índice nos permite, otra vez más, afirmar que la sociedad catalana es más igualitaria, como así se muestra en el ámbito laboral y, por ello, más moderna que la sociedad andaluza.

Los valores ofrecidos por la dimensión de participación laboral ofrecen interesantes contrastes entre las dos comunidades aquí estudiadas. De nuevo, observamos que Cataluña $(0,221)$ es la comunidad donde existe menor nivel de desigualdad entre los géneros en el ámbito laboral y, en este caso concreto, en torno a la participación en el trabajo. El valor del índice en esta dimensión para el caso de Cataluña es incluso inferior al de España. Sin embargo, el caso de Andalucía es justo el contrario, su valor excede al de la media española y se aleja en mayor medida del 0 , supuesta situación de igualdad. Además, como han observado Bericat y Sánchez (2008: 10), se puede pensar que la participación laboral de las mujeres en nuestro país parece depender en mayor medida del tipo de sistema económico-productivo de cada comunidad autónoma, mientras que la desigualdad en las condiciones de trabajo parece explicarse por factores de carácter estructural que operan con altos niveles de homogeneidad, como así se refleja en los datos de la última columna de la tabla 4 (los datos de la desigualdad en las condiciones de trabajo son bastante parecidos en los tres contextos observados).

\section{Factores personales y mercado de trabajo}

\subsection{La actividad laboral de la mujer casada según el prestigio ocupacional del marido o compañero}

Existen diferencias entre los grupos de las mujeres casadas que trabajan fuera de casa y las que no respecto al prestigio ocupacional de sus maridos. En la tabla 5, se contempla, a través del test de diferencias de medias, que las disimilitudes observadas en el nivel de prestigio de los maridos de las mujeres que pertenecen a los dos grupos aquí considerados (mujeres que trabajan fuera de casa y mujeres que no trabajan fuera) son estadísticamente significativas.

Montoro (2003: 187) muestra que, a lo largo del siglo XX, la posición social del marido ha determinado la posición relativa de la mujer en España ${ }^{16}$. La autora citada constata el hecho de que, a principios del siglo XX, en España, cuanto más alta era la clase social del varón (marido), más baja resultaba la probabilidad de que la mujer (esposa) trabajase fuera de casa. Asimismo, observa la importante transformación que se vive en nuestro país sobre todo a partir de la segunda mitad de los años setenta, cuando, además de aumentar con- 
Tabla 5. Prestigio ocupacional del marido o compañero según la situación profesional de la mujer

\begin{tabular}{|c|c|c|c|c|c|c|c|c|c|}
\hline & \multicolumn{3}{|c|}{ Andalucía (2) } & \multicolumn{3}{|c|}{ Cataluña (3) } & \multicolumn{3}{|c|}{ España (1) } \\
\hline & $\begin{array}{l}\text { Trabaja } \\
\text { fuera de } \\
\text { casa (a) }\end{array}$ & $\begin{array}{c}\text { No } \\
\text { trabaja } \\
\text { fuera (b) }\end{array}$ & $\begin{array}{l}\text { Dif. } \\
a-b\end{array}$ & $\begin{array}{l}\text { Trabaja } \\
\text { fuera de } \\
\text { casa (a) }\end{array}$ & $\begin{array}{c}\text { No } \\
\text { trabaja } \\
\text { fuera (b) }\end{array}$ & $\begin{array}{l}\text { Dif. } \\
\text { a-b }\end{array}$ & $\begin{array}{l}\text { Trabaja } \\
\text { fuera de } \\
\text { casa (a) }\end{array}$ & $\begin{array}{c}\text { No } \\
\text { trabaja } \\
\text { fuera (b) }\end{array}$ & $\begin{array}{l}\text { Dif. } \\
\text { a-b }\end{array}$ \\
\hline \multicolumn{10}{|l|}{ Media } \\
\hline Prestigio & 116,25 & 100,75 & $15,49^{* * *}$ & 115,08 & 104,35 & $10,72^{* * *}$ & 116,61 & 105,571 & $11,04^{* * *}$ \\
\hline $\mathrm{N}$ & 468 & 1768 & & 798 & 1385 & & 3968 & 9260 & \\
\hline
\end{tabular}

Fuente: Encuesta de Calidad de Vida en el Trabajo 1999-2004.

*** Estadístico de la prueba t de diferencia de medias para muestras independientes.

siderablemente el porcentaje de mujeres casadas que trabajan, también cambia la relación entre el trabajo de la mujer y la clase social del marido. Así, a partir de este punto de inflexión, cuanto más alta es la clase social del marido, más trabaja la mujer fuera de casa (el porcentaje de este grupo es incluso superior al de las mujeres que pertenecen a la clase obrera). Montoro (2003: 189) llega a la conclusión siguiente:

Cuanto más nos acercamos a una sociedad moderna, los individuos mejor situados socialmente tienen esposas trabajadoras, o, desde otra perspectiva, las mujeres trabajadoras están casadas con hombres bien situados, en una proporción significativamente superior a las mujeres amas de casa ${ }^{17}$.

Y además, conforme han destacado Alberdi, Escario y Matas (2000), en la década de 1990, se han invertido los papeles: trabajan fuera del hogar —en una proporción significativamente superior - las mujeres que tienen una buena posición social y un elevado nivel de estudios. Desde el punto de vista del matrimonio, las mujeres casadas con hombres pertenecientes a los estratos sociales medio-altos trabajan fuera del hogar más que las mujeres casadas con hombres pertenecientes a los estratos sociales inferiores. De manera que el permanecer en el hogar y ser exclusivamente ama de casa no es una tarea que goce de prestigio social.

Los resultados obtenidos en el siguiente análisis muestran que las mujeres trabajadoras están casadas con hombres bien situados, esto es así tanto para España como para las dos comunidades analizadas. En ambas comunidades (Andalucía y Cataluña), las medias del prestigio ocupacional de los esposos de las mujeres que trabajan fuera de casa son mayores a las de aquellas esposas que ejercen de amas de casa.

Las diferencias de prestigio ocupacional de los maridos o compañeros entre los grupos de mujeres que trabajan fuera de casa y de las que no trabajan fuera son estadísticamente significativas (véase la tabla 5). 
Resulta interesante observar que, en Andalucía, dicha diferencia $\left(15,49^{* * *}\right)$ es mayor que en Cataluña $\left(10,72^{* * *}\right)$. Además, las diferencias de medias de prestigio ocupacional de los maridos de ambos grupos de mujeres en Andalucía supera la media española $\left(11,04^{* * *}\right)$ en 4,45 puntos. Si bien la diferencia de medias observada en Cataluña es incluso menor que la media española.

Los datos obtenidos indican que Cataluña se halla más cerca de la idea de «modernización» aquí planteada (que las categorías sociales pierden protagonismo en la explicación de las conductas de los individuos) que Andalucía. La distancia entre las medias de los prestigios de los hombres que están casados con mujeres de distinta situación laboral (trabajando fuera de casa / no trabajando fuera de casa) es menor en Cataluña que en Andalucía, lo que permite considerar que, en Cataluña, categorías sociales como el prestigio ocupacional del marido o compañero ejercen menos presión sobre la actividad laboral de la mujer, con lo que se acerca más al modelo de sociedad moderna, caracterizada por el creciente proceso de individuación.

\subsection{La probabilidad de que la mujer casada trabaje fuera de casa en Andalucía y Cataluña}

Como se indicó previamente en el apartado dedicado a la metodología con el objeto de precisar las categorías sociales (de la mujer y del marido o compañero) que contribuyen a situar a las mujeres dentro del mercado laboral, se han estimado tres modelos de regresión logística logit: uno para el conjunto de España (a través del cual se comprueba que, del conjunto del total de regiones españolas, las mujeres casadas catalanas son las que tienen mayor probabilidad de trabajar fuera de casa) ${ }^{18}$ y otros dos para cada una de las comunidades autónomas aquí analizadas ${ }^{19}$. En este análisis, se han utilizado los datos recogidos por la Encuesta PHOGUE en el año 2000. Se trata de una encuesta realizada por el INE con una muestra ampliada que resulta significativa a nivel de las comunidades autónomas. La variable «dependiente» es la actividad de la mujer, que toma el valor 1 cuando la mujer casada trabaja fuera de casa y 0 en caso contrario (variable dicotómica).

En dicho modelo, se han incluido como variables independientes, referidas, por un lado, a las de las mujeres casadas, las siguientes: la edad (dividida en cinco categorías: de 18 a 24 años, de 25 a 34, de 35 a 44, de 45 a 54, de 55 a 64), el nivel educativo (dividido en siete categorías: analfabetos, con estudios primarios, con estudios de primer ciclo de secundaria, con estudios de FPI, con estudios de FPII, con educación de segundo ciclo de secundaria, con estudios universitarios de ciclo corto, con estudios universitarios de ciclo largo), la discapacidad (sin discapacidad, con discapacidad intensa, con cierta discapacidad, con discapacidad que no impide desarrollar sus actividades diarias), la

18. Véase este modelo en la tabla incluida en el anexo.

19. Según datos del PHOGUE 2000, la tasa de mujeres casadas que trabaja quince horas semanales o más fuera de casa es del 46,1\% en Cataluña y del 25,9\% en Andalucía. 
tenencia de hipoteca (dicotómica: no tiene hipoteca, tiene hipoteca), la dedicación empleada al cuidado de los niños (cuida a niños, no cuida a niños) y de adultos dependientes (también dividida en dos categorías: cuida y no cuida), y, por otro lado, las variables que hacen referencia a características de los maridos o compañeros: el nivel educativo (se le ha dado el mismo tratamiento que al grupo de mujeres), la situación profesional (en tres categorías: no trabaja, asalariado, autónomo) y los ingresos obtenidos en el año anterior (en pesetas, al ser la referencia temporal del año 1999).

Se ha seleccionado como mujer casada de referencia aquella de edad de 18 a 24 años, sin estudios, que no tiene discapacidad, sin hipoteca sobre su vivienda, que no cuida niños ni adultos y cuyo marido no trabaja y es analfabeto.

La tabla 6 presenta los resultados de la estimación de los modelos logit para la probabilidad de que las mujeres casadas que viven en Andalucía y Cataluña trabajen fuera de casa ${ }^{20}$. Los estadísticos generales que figuran en su parte inferior señalan que, en ambos casos, los modelos son significativos en su conjunto (Prob $>$ chi $2=0.0000$ ) y que la bondad del ajuste es aceptable (Pseudo $\mathrm{R} 2=21,37 \%$ para Andalucía y 19,09\% para Cataluña).

Las estimaciones muestran que, en Andalucía, a más edad de la mujer casada, menor probabilidad de que ésta trabaje fuera de casa (la probabilidad de que las mujeres casadas entre 55 y 64 años trabajen fuera de casa es 11,38 puntos porcentuales inferior a que lo haga una de 18 a 24 años, y esta relación es significativa al 15\%). En contraste, en Cataluña, la edad no influye sobre la probabilidad de que las mujeres casadas trabajen fuera.

En Andalucía, un nivel educativo más elevado de la mujer casada está asociado a una mayor posibilidad de que ésta tenga un trabajo remunerado en mayor proporción que en Cataluña. La probabilidad de que la mujer casada andaluza con estudios universitarios cortos y largos trabaje es, respectivamente, de 68,10 y 64,68 puntos porcentuales superior a la de la casada analfabeta. En Cataluña, la probabilidad de que la mujer casada con estudios universitarios largos trabaje es del 54,14\% y la de las que cuentan con estudios universitarios cortos, del $41,84 \%$. Como se puede apreciar, estas probabilidades son inferiores a las de la comunidad autónoma andaluza. De ello se desprende que el peso de esta variable también es menor en Cataluña.

En Andalucía, la relación entre la tenencia de una hipoteca y la probabilidad de que la mujer casada trabaje fuera de casa es estadísticamente significativa al $1 \%$, es decir, afecta mucho y positivamente al hecho de que la mujer casada trabaje fuera de casa. Sin embargo, en Cataluña, esta relación goza de una significación estadística más tenue (el 15\%).

Tanto en Andalucía como en Cataluña, el tener que cuidar de niños y/o de adultos ejerce un efecto negativo en la probabilidad de que las mujeres casa-

20. Se han eliminado de la muestra las mujeres que trabajan menos de quince horas semanales y aquéllas con edad igual o superior a sesenta y cinco años. 
das trabajen fuera de casa ${ }^{21}$. En otras palabras, el tener que cuidar de dependientes contribuye a apartar a las mujeres casadas del mercado laboral. Lo que resulta interesante es comprobar que, en Cataluña, la probabilidad de que la mujer casada con la obligación de cuidar de niños entre sus tareas diarias trabaje es inferior a la de la que no cuida niños es de 16,14 puntos porcentuales. En contraste, en Andalucía, las mujeres que están en esa misma situación, que están casadas y cuidan de niños, tienen una probabilidad 65,89 puntos porcentuales menor de trabajar que las que no cuidan de niños. De lo que parece desprenderse que el cuidado de los niños es un importante obstáculo para que las mujeres casadas tengan un empleo remunerado en Andalucía y no tanto en Cataluña.

Por otro lado, la probabilidad de no trabajar de la casada catalana que cuida de adultos $(21,75 \%)$ es superior a la de la andaluza $(12,75 \%)^{22}$. El cuidado de los adultos pesa más en la probabilidad de trabajar de estas mujeres en Cataluña que en Andalucía.

La influencia del nivel educativo del marido o compañero en la probabilidad de que la mujer casada "trabaje», resulta también de gran interés, pues guarda una estrecha relación con la tesis defendida a lo largo de este artículo. Recordemos la teoría de Montoro, que presenta como rasgo propio de cambio social y de modernidad la transformación en la tendencia de la relación entre el estatus ocupacional del marido y la situación laboral de la mujer, afirmando que si durante mucho tiempo en España se observa que, a mayor estatus ocupacional del marido, menor probabilidad de que la mujer tenga un trabajo remunerado, el proceso de modernización consigue invertir la relación, de tal forma que, según los datos del PHOGUE de 1999, se puede observar que, a mayor estatus ocupacional del marido, mayor probabilidad de la mujer casada de trabajar fuera ${ }^{23}$. Pues bien, este fenómeno se aprecia en Cataluña pero no en Andalucía. En esta última comunidad, y siempre según los resultados del modelo presentado, no se observa relación entre el nivel educativo de los maridos con estudios universitarios y la probabilidad de trabajar fuera de casa de la mujer casada, mientras que en Cataluña la relación es positiva. Así, en el caso de que la mujer esté casada con un hombre con estudios de nivel univer-

21. Un análisis logit realizado previamente al aquí presentado (y que no mostramos por limitaciones de espacio) ya indicaba la fuerte relación inversa existente entre el número de horas dedicado al cuidado de dependientes y la probabilidad de la mujer casada de trabajar fuera de casa. A su vez, dejaba entrever el complicado fenómeno de la conciliación familiar y laboral, axí como su incidencia en el desempeño del rol laboral femenino.

22. El interés que puedan suscitar estos datos son merecedores de un futuro trabajo que abarque en profundidad el tema de la conciliación familiar y laboral, así como los cambios habidos tras la puesta en vigor de la Ley 39/1999, de 5 de noviembre, para promover la conciliación de la vida familiar y laboral de las personas trabajadoras; la Ley 39/2006, de 14 de diciembre, de promoción de la autonomía personal y atención a las personas en situación de dependencia, y la Ley Orgánica 3/2007, de 22 de marzo, para la igualdad efectiva de mujeres y hombres.

23. Relacionado con este hecho, se encuentra la tendencia actual de las sociedades modernas hacia la homogamia. Véase Mayoral y Samper (2006). 
Tabla 6. Estimaciones LOGIT de la probabilidad de que la mujer casada trabaje fuera de casa. Andalucía y Cataluña (1999)

\begin{tabular}{|c|c|c|c|c|}
\hline \multirow[b]{3}{*}{ Variables } & \multicolumn{4}{|c|}{ Variable dependiente: trabaja más de 15 horas } \\
\hline & \multicolumn{2}{|c|}{ CA Andalucía } & \multicolumn{2}{|c|}{ Cataluña } \\
\hline & Coef. & E. marg. & Coef & E. marg. \\
\hline Constante & $-2,0275$ & & $-1,3033$ & \\
\hline \multicolumn{5}{|l|}{ Edad de la mujer: } \\
\hline $25-34$ & 0,1172 & 0,0187 & 0,4804 & 0,1194 \\
\hline $35-44$ & 0,6857 & 0,1155 & 0,5393 & 0,1339 \\
\hline $45-54$ & 0,2066 & 0,0334 & 0,4258 & 0,1058 \\
\hline $55-64$ & $-0,8555^{\wedge}$ & $-0,1138$ & $-0,6607$ & $-0,1582$ \\
\hline \multicolumn{5}{|c|}{ Nivel de estudios de la mujer: } \\
\hline Primarios & $1,2043^{* * *}$ & 0,2171 & 0,5066 & 0,1258 \\
\hline Secundarios primer ciclo & $1,3704^{* * *}$ & 0,2505 & $0,8152^{*}$ & 0,2009 \\
\hline Secundarios segundo ciclo & $1,8159^{* * *}$ & 0,3953 & $1,5971^{* * *}$ & 0,3642 \\
\hline FP I & $2,2058^{* * *}$ & 0,4858 & $1,4308^{* * *}$ & 0,3310 \\
\hline FP II & $1,9345^{* * *}$ & 0,4231 & $1,6815^{* * *}$ & 0,3718 \\
\hline Universitarios cortos & $3,3256^{* * *}$ & 0,6810 & $1,9802^{* * *}$ & 0,4184 \\
\hline Universitarios largos & $3,0809^{* * *}$ & 0,6468 & $3,2180^{* * *}$ & 0,5415 \\
\hline Tenencia de hipoteca & $0,9281^{* * *}$ & 0,1610 & $0,2622^{\wedge}$ & 0,0651 \\
\hline \multicolumn{5}{|l|}{ Cuidado de dependientes: } \\
\hline Cuida niños & $-0,4266^{* *}$ & $-0,6589$ & $-0,6618^{* * *}$ & $-0,1614$ \\
\hline Cuida adultos & $-1,1557^{* * *}$ & $-0,1275$ & $-0,9711^{* * *}$ & $-0,2175$ \\
\hline Cuida niños y adultos & $-0,0470$ & $-0,0072$ & $-0,4057$ & $-0,0976$ \\
\hline \multicolumn{5}{|c|}{ Situación laboral del marido o compañero: } \\
\hline Asalariado & 0,2247 & 0,0348 & 0,1761 & 0,0435 \\
\hline Autónomo & 0,1308 & 0,0210 & 0,1091 & 0,0271 \\
\hline \multicolumn{5}{|c|}{ Nivel educativo del marido o compañero: } \\
\hline Primarios & $-0,8294^{* * *}$ & $-0,1153$ & 0,2205 & 0,0548 \\
\hline Secundarios primer ciclo & $-0,4440^{\wedge}$ & $-0,0652$ & 0,0806 & 0,0200 \\
\hline Secundarios segundo ciclo & $-0,2188$ & $-0,0323$ & 0,4510 & 0,1122 \\
\hline FP I & $-0,9544^{* * *}$ & $-0,1130$ & 0,5581 & 0,1385 \\
\hline FP II & $-0,6689^{*}$ & $-0,8696$ & 0,2769 & 0,0690 \\
\hline Universitarios cortos & $-0,3394$ & $-0,0484$ & $0,7232^{\wedge}$ & 0,1780 \\
\hline Universitarios largos & $-0,0403$ & $-0,0062$ & $1,0664^{*}$ & 0,2569 \\
\hline Ingresos por 100.000 ptas. & $-0,0303^{* * *}$ & $-0,0047$ & $-1,3033^{* *}$ & $-0,0032$ \\
\hline \multicolumn{3}{|c|}{ Número de observaciones 1.061} & \multicolumn{2}{|l|}{890} \\
\hline \multicolumn{3}{|c|}{$\begin{array}{ll}\mathrm{L} \text { chi2 }(28) & 260.40\end{array}$} & \multicolumn{2}{|l|}{234.43} \\
\hline Prob > chi2 & 0.0000 & \multicolumn{2}{|r|}{0.0000} & \\
\hline Pseudos R2 & \multicolumn{2}{|l|}{0.2137} & \multicolumn{2}{|l|}{0.1909} \\
\hline
\end{tabular}

Fuente: Panel de Hogares de la Unión Europea (PHOGUE), 1999. Muestra ampliada.

${ }^{* * *}$ indica significatividad estadística al $1 \%,{ }^{* *}$ al $5 \%,{ }^{*}$ al $10 \%, \wedge$ al $15 \%$. 
sitario largo, la probabilidad de ésta de trabajar fuera es 25,69 puntos porcentuales superior a la de la casada con un analfabeto.

Además, en Andalucía, el nivel educativo del varón (compañero o marido) para los niveles más bajos del sistema pesa negativamente sobre la probabilidad de que la mujer casada trabaje fuera de casa (hasta el grado de secundaria, de segundo ciclo, a mayor nivel educativo del marido, menor probabilidad de que la mujer casada trabaje).

A medida que crecen los ingresos del marido, menor es la probabilidad de que las mujeres casadas en Andalucía trabajen fuera de casa. Esta probabilidad es mayor en Andalucía (0,47\%) que en Cataluña (0,32\%), donde, a su vez, la relación es estadísticamente menos significativa.

En resumen, en Cataluña, la probabilidad de que la mujer casada trabaje fuera de casa no está determinada por la edad de ésta. Y viene explicada en menor medida que en Andalucía por el nivel de estudios de la mujer, los ingresos y la responsabilidad de tener que cuidar niños. Si bien sí existe una relación positiva entre la probabilidad de trabajar fuera de la mujer casada catalana con un varón de estudios universitarios largos.

\section{Conclusiones}

La evolución vivida en Andalucía y Cataluña en el ámbito de la estructura laboral en el período que va de 1995 a 2005 es la de un cambio hacia una sociedad más igualitaria en las cuestiones de género, y por ello se avanza hacia una sociedad más moderna. Incluso en algunos casos se ha podido observar que ambas comunidades adquieren posiciones de mayor avance en el proceso de modernización que el conjunto nacional.

No obstante, el análisis efectuado permite observar importantes diferencias en materia de desigualdad sexual en el ámbito laboral en las dos regiones estudiadas (Andalucía y Cataluña). Se constata la hipótesis de partida que sostenía que los dos contextos objeto de análisis son estructuralmente diferentes. Se ratifica, a través de los datos, que Cataluña se encuentra en una posición más cercana a la modernización en el plano laboral y siempre desde la perspectiva de género. Así, por ejemplo, esta comunidad ofrece para las mujeres mayor tasa de actividad y empleo y menor tasa de paro femenino, según los datos de 2005 (EPA segundo trimestre).

Cataluña vive un proceso de acercamiento a la igualdad de los sexos en el ámbito estructural laboral más rápido que Andalucía (1995-2005). Entre otras razones, porque Cataluña experimenta un incremento de la tasa de actividad y de empleo femenino superior a la masculina. Sin embargo, en Andalucía todavía esto no es así, aunque se observa el comienzo de esta nueva tendencia en los datos del empleo.

Se constata que el prestigio ocupacional del marido de las mujeres que trabajan fuera de casa es mayor del de aquellos maridos cuyas mujeres no trabajan fuera del hogar (tesis mantenida por Montoro, 2003). Se ratifica el hecho de que las mujeres trabajadoras están casadas en mayor proporción con hom- 
bres de mayor prestigio ocupacional que las mujeres que se dedican en exclusiva a las tareas propias del hogar. En Cataluña, es menor la influencia del capital colectivo (operativizado en este caso a través de la relación entre la situación laboral de la mujer y el prestigio ocupacional del marido) que en Andalucía. Como resultado, se concluye que Cataluña se encuentra más cercana al proceso de individuación fruto de la modernización.

Los análisis a través de tres modelos logit han permitido precisar qué categorías sociales (de la mujer y del marido o compañero) contribuyen a situar a las mujeres casadas, catalanas y andaluzas, dentro del mercado de trabajo.

Se comprueba que la probabilidad de la mujer casada catalana de trabajar fuera de casa está menos determinada que la andaluza por ciertos condicionantes como la edad (que, en el caso de Cataluña, no ejerce influencia), el nivel de estudios personal, la responsabilidad de cuidados de niños e ingresos, entre otros. Estos datos permiten situar a la comunidad catalana más cerca del modelo de modernización descrito.

En contraste, en Andalucía, todas las variables incluidas en el análisis influyen en la probabilidad de que la mujer casada trabaje: la edad y el nivel de estudios de la mujer, el tener que cuidar de niños, el tener que cuidar de adultos y el nivel educativo del marido (siempre que éste no supere los estudios universitarios), a excepción de la situación laboral del marido o compañero (asalariado, autónomo), que no ejerce influencia en ninguna de las dos comunidades autónomas. Estos resultados llevan a situar a la comunidad andaluza en un punto más alejado del modelo de modernización aquí utilizado.

También es interesante destacar que el análisis logit ha permitido volver a verificar la afirmación de que, en las sociedades más avanzadas, se produce un cambio en el comportamiento laboral de las mujeres, de manera que, cuanto más alta es la clase social del marido, más trabaja la mujer fuera de casa (desarrollada en el apartado 4.1.). Este hecho se comprueba para el caso de Cataluña y no para el de Andalucía. En Cataluña, las mujeres casadas con maridos con estudios universitarios tienen una probabilidad de trabajar fuera de casa de 25,69 puntos porcentuales mayor que las casadas con analfabetos. Sin embargo, en Andalucía se observa que todavía se mantiene fiel al comportamiento que ha prevalecido a lo largo de principios del siglo XX, con lo que se confirma que cuanto más alta era la clase social del marido, más baja resultaba la probabilidad de que la mujer trabajase fuera del hogar.

En resumen, tanto los indicadores estructurales como el análisis de la influencia de los factores personales y de la pareja en el hecho de que la mujer casada trabaje fuera del hogar, permiten situar a Cataluña más cerca de la consecución de la idea de modernización aquí desarrollada. 


\section{Referencias bibliográficas}

Alberdi, I.; Escario, P. y MATAs, N. (2000). Las mujeres jóvenes en España. Barcelona: Fundación La Caixa. Colección Estudios Sociales, 4.

BECK, U. (2000). Un nuevo mundo feliz: La precariedad del trabajo en la era de la globalización. Barcelona: Paidós.

Bericat Alastuey, E. y Sánchez Bermejo, E. (2008). «Balance de la desigualdad de género en España». Revista Actual, 23. Sevilla: Centro de Estudios Andaluces. Consejería de la Presidencia.

- (2003). "Fragmentos de la realidad social posmoderna». Revista Española de Investigaciones Sociológicas, 102, abril-junio, 9-45.

Bernstein, R.J. (ed.) (1985). Habermas and Modernity. Londres: Polity Press.

Carabaña, J. y Gómez Bueno, C. (1996). Escalas de prestigio ocupacional. Madrid: CIS.

Carbonero Gamundi, M.A. (1997). Estrategias laborales de las familias en España. Madrid: Consejo Económico y Social. Colección Estudios.

Castaño Collado, C. (dir. y coord.) (2002). Indicadores laborales básicos de la situación de la mujer en España y sus regiones. Proyecto realizado para el Instituto de la Mujer por el Laboratorio de Investigación del sector Servicios (SERVILAB).

Díez Nicolás, J. (2006). «Prólogo». En: InglegharT, R. y Welzel, Ch. Modernización, cambio cultural y democracia: la secuencia del desarrollo humano. Madrid: CIS, IX-XIII.

Dolton, P.; MARCENADO, O. y SKAll, A. (2008). "Gender differences across Europe». En: BetTIO F. y VerashCHAGINA (eds.). Frontiers in the economics of gender. Londres: Rouledge Siena Studies in Political Economy.

ECHEVERRÍA, J. (1997). "La movilidad social de las mujeres y el problema de la unidad de análisis de clase». Revista Internacional de Sociología (RIS), 17, 83-116.

EisenstadT, S.N. (1964). «Breakdowns of modernization». Economic Development and Cultural Change, 25, supplement, 67-98.

Esping Andersen, G.; GÜEll, M. y Brodmann, S. (2005). «When mothers work and fathers care. Joint household fertility decision making». Demosoc Working Paper, 4. Universitat Pompeu Fabra

Feito, R. (1995). «Mujeres y análisis de clases». Revista Española de Investigaciones Sociológicas, 69, 149-171.

GAMERO, C. (2005). Análisis microeconómico de la satisfacción laboral. Madrid: Consejo Económico y Social.

GARRIDO, L. (1992). Las dos biografías de la mujer en España. Madrid: Instituto de la Mujer.

GIDDENS, A. (1991). Modernity and self-identity. Standford: Standford University Press.

Giner, S.; Lamo de Espinosa, E. y TORres, C. (eds.). (1998): Diccionario de sociología. Madrid: Alianza Editorial.

GobernADO ARRIBAS, R. (1994). «Modernidad y estratificación social: análisis comparativo de las estructuras sociales de Cataluña y Andalucía». Revista Española de Investigaciones Sociológicas, 67, julio-septiembre, 87-108.

- (1996a). "Los límites de la expansión educativa». Revista Española de Pedagogía, 205, 487-510.

- (1996b). «El logro ocupacional en España veinte años después». Revista Española de Investigaciones Sociológicas, 74, 209-224.

- (1996c). Análisis comparado de las estructuras sociales en Andalucía y Cataluña. Málaga: Estudios y Ensayos. Universidad de Málaga y Universidad de Almería. 
- (2003). «El logro ocupacional como indicador de organización de la familia». En: Caparrós, N.; LÓPEZ, D. y Montoro, C. (2003): Implicaciones del cambio social en la familia. Navarra. Universidad de Navarra. Instituto de Ciencias de la Familia, 135-151.

GolDTHORPE, J.H. (2007). «Globalisation and social class». En: On Sociology. Standford, California: Stanford University Press, I, 91-116.

INGLEHART, R. y WELZEL, Ch. (2006). Modernización, cambio cultural y democracia: La secuencia del desarrollo humano. Madrid: Centro de Investigaciones Sociológicas.

MAYORAL, D. y SAMPER, L. (2006). "Cambio social y homogamia educativa». Revista Internacional de Sociología, LXIV, 43, enero-abril, 35-67.

Miguel, J. de (1998). Estructura y cambio social en España. Madrid: Alianza.

Montoro Gurich, C. (2003). «Cambios en el estatus social de las mujeres casadas en la España del siglo XX». Revista Española de Investigaciones Sociológicas, 104, 181-198.

PARSONS, T. (1955): Family, socialization and interaction process. Glencoe, Ill.: Free Press.

PHOGUE, Panel de Hogares de la Unión Europea (2000), Eurostat.

REQUENA SANTOS, F. (2005). La estructura ocupacional española. Madrid: Ministerio de Trabajo y Asuntos Sociales.

SALIDO, O. (1998). El proceso de movilildad: diferencias y peculiaridades de las mujeres y los hombres. Texto inédito presentado en el VI Congreso Español de Sociología, La Coruña.

- (2007). «La incorporación de las mujeres al empleo: Hacia un nuevo contrato social». En: CARbonero, M.A. y LeVIN, S. (coord.). Trabajo, familia y estado: Las transformaciones en las relaciones de género. Rosario, Argentina: Homo Sapiens.

SMELSER, N. (1992). "Hacia una teoría de la modernización». En: ETZIONI, A.y ETZIONI, E. (eds.). Los cambios sociales: fuentes, tipos y consecuencias. México: Fondo de Cultura Económica, 235-249.

Solé, C. (1987). Ensayos de teoría sociológica: Modernización y postmodernización. Madrid: Paraninfo.

TeZANOS, J.F. (1984). «Cambio social y modernización en la España actual». Revista Española de Investigaciones Sociológicas, 28, 19-62.

Товío, C. (1994). "El acceso de las mujeres al trabajo, el espacio y el urbanismo». Boletín Especial Mujer y Ciudad. [htpp://habitat.aq.upm.es/boletin/n7/actob.html]

TREIMAN, D.J. (1970). «Industrialization and social stratification». En: LAUMANN, E. (ed.). Social stratification: Research and theory for the 1970's. Nueva York: The Bobbs-Merril Company, 207-234.

Welzel, Ch.; Inglehart, R. y Klingemann, H.D. (2003). "The theory of human development: A cross-cultural analysis». European Journal of Political Research, 42, 341-379. 


\section{Anexo}

Tabla 7. Estimaciones logit de la probabilidad de que la mujer casada travaje fuera de casa. España (1999)

\begin{tabular}{|c|c|c|}
\hline Variables & Coef & E. marg. \\
\hline Constante & $-1,2993$ & \\
\hline \multicolumn{3}{|l|}{ Edad de la mujer: } \\
\hline $25-34$ & 0,1624 & 0,0365 \\
\hline $35-44$ & $0,2987^{\wedge}$ & 0,0675 \\
\hline $45-54$ & $-0,0647$ & $-0,0142$ \\
\hline $55-64$ & $-0,7653^{* * *}$ & $-0,1554$ \\
\hline \multicolumn{3}{|l|}{ Nivel de estudios de la mujer: } \\
\hline Primarios & $0,2786^{* *}$ & 0,0629 \\
\hline Secundarios primer ciclo & $0,5141^{* * *}$ & 0,1179 \\
\hline Secundarios segundo ciclo & $1,0969^{* * *}$ & 0,2637 \\
\hline FP I & $1,1580^{* * *}$ & 0,2794 \\
\hline FP II & $1,4007^{* * *}$ & 0,3360 \\
\hline Universitarios cortos & $2,3490^{* * *}$ & 0,5188 \\
\hline Universitarios largos & $2,4122^{* * *}$ & 0,5266 \\
\hline Tenencia de hipoteca & $0,4144^{* * *}$ & 0,0942 \\
\hline \multicolumn{3}{|l|}{ Cuidado de dependientes: } \\
\hline Cuida niños & $-0,5953^{* * *}$ & $-0,1128$ \\
\hline Cuida adultos & $-0,4960^{* * *}$ & $-0,1007$ \\
\hline Cuida niños y adultos & $-0,6911^{* * *}$ & $-0,1337$ \\
\hline \multicolumn{3}{|c|}{ Situación laboral del marido o compañero: } \\
\hline Asalariado & $0,2931^{* * *}$ & 0,0644 \\
\hline Autónomo & $0,4931^{* * *}$ & 0,1141 \\
\hline \multicolumn{3}{|c|}{ Nivel educativo del marido o compañero: } \\
\hline Primarios & $-0,2149^{*}$ & $-0,0469$ \\
\hline Secundarios primer ciclo & $-0,1412$ & $-0,0309$ \\
\hline Secundarios segundo ciclo & $-0,0419$ & $-0,0092$ \\
\hline FP I & $-0,0783$ & $-0,0171$ \\
\hline FP II & $-0,1270$ & $-0,0276$ \\
\hline Universitarios cortos & 0,0806 & 0,0181 \\
\hline Universitarios largos & $0,3701^{* *}$ & 0,0858 \\
\hline $\begin{array}{l}\text { Ingresos por } 100.000 \text { ptas } \\
\text { CCAA: }\end{array}$ & $-0,0155^{* * *}$ & $-0,0034$ \\
\hline Asturias & 0,2042 & 0,0466 \\
\hline Baleares & $0,6298^{* * *}$ & 0,1502 \\
\hline Canarias & $0,3766^{* *}$ & 0,0876 \\
\hline Cantabria & $0,2266^{\wedge}$ & 0,0519 \\
\hline Castilla-La Mancha & $-0,0527$ & $-0,0116$ \\
\hline Castilla y León & 0,1074 & 0,0242 \\
\hline Cataluña & $0,7515^{* * *}$ & 0,1785 \\
\hline Valencia & $0,4298^{* * *}$ & 0,1003 \\
\hline Extremadura & $-0,3585^{* * *}$ & $-0,0748$ \\
\hline Galicia & $0,6971^{* * *}$ & 0,1661 \\
\hline Madrid & $0,2854^{* *}$ & 0,0656 \\
\hline
\end{tabular}


Tabla 7. Estimaciones logit de la probabilidad de que la mujer casada travaje fuera de casa. España (1999) (continuación)

\begin{tabular}{lcc}
\hline Variables & Coef & E. marg. \\
\hline Murcia & $0,2246^{*}$ & 0,0514 \\
Navarra & $0,3960^{* *}$ & 0,0925 \\
País Vasco & 0,1999 & 0,0456 \\
\hline Número de observaciones & 7.872 & \\
\hline LR chi2 $(42)$ & 1767,29 & \\
\hline Prob > chi2 & 0,0000 & \\
\hline Pseudo s $\mathbf{R}^{2}$ & 0,1716 & \\
\hline
\end{tabular}

Fuente: Panel de Hogares de la Unión Europea (PHOGUE), 1999. Muestra ampliada.

${ }^{* * *}$ indica significatividad estadística al $1 \%,{ }^{* *}$ al $5 \%,{ }^{*}$ al $10 \%, \wedge$ al $15 \%$. 